\title{
The influence of the weather conditions on main atmospheric pollutants concentration in Chengdu*
}

\author{
Qin Liu ${ }^{\mathrm{a}}$, Zeng-Wu Wang ${ }^{\mathrm{a}}$, Qian-Ru Ye ${ }^{\mathrm{a}}$, De-Ping Zhou ${ }^{\mathrm{b}}, \mathrm{Xu}$ Deng ${ }^{\mathrm{a}}$, \\ Lei Hua and Hong-Ling Yin a, ${ }^{\dagger}$ \\ ${ }^{a}$ College of Resources and Environment, \\ Chengdu University of Information Technology, \\ Chengdu, Sichuan 610225, China, \\ ${ }^{b}$ Environmental Quality Supervision Station of Chengdu Military Region, \\ Chengdu, Sichuan, 610041, China, \\ E-mail: yhl@cuit.edu.cn \\ Phone No. of correspondence author: $+86135-41352807$
}

\begin{abstract}
Base on the meteorological data and the monitoring data $(28 / 10 / 2013 \sim 16 / 5 / 2014)$ of the main atmospheric pollutants, $\mathrm{PM}_{2.5}, \mathrm{PM}_{10}, \mathrm{CO}, \mathrm{NO}_{2}$ and $\mathrm{SO}_{2}$ of Chengdu, the comparative analysis of the relationship between the pollutant concentrations and meteorological conditions of Chengdu were carried out. The influence of meteorological conditions change on the atmospheric pollutants concentration was also discussed Results showed that the air pollution of Chengdu is more serious when the wind is from southern area than the wind from the northern area. Pollutant concentrations were positively correlated with wind speed and humidity, and the outside sources is covering $10 \% \sim 40 \%$ when the wind is strong. Clearance ratio of rainfall to Chengdu air pollutants can reach more than $60 \%$, and the scouring effect can reduce $40 \% \sim 60 \%$ of the particulate matter. The influence of the temperature on the atmospheric pollution is mainly embodied in temperature inversion during the different seasons influencing the diffusion of the atmospheric pollutants. It is more conducive for pollutant diffusion on overcast days than cloudy days.
\end{abstract}

Keywords: Weather Conditions; Atmospheric Pollution; Chengdu city.

\section{Introduction}

With the rapid increasing of Chinese urbanization and energy consumption, the changes of atmospheric environment took place which leaded to the changes of atmospheric environmental quality, and brought a lot of atmospheric pollution problems $[1,2]$. To solve the atmospheric pollution problems, the study of the

\footnotetext{
* We acknowledge financial support from National Natural Science Youth Fund (21407014), Science and Technology Support Program of Sichuan Province (2015GZ0240), Key projects of Sichuan Provincial Department of Education (13Z197), the Key Laboratory Fund for Atmospheric Pollution Control and Environmental Simulation of Chengdu University of Information Technology (KFKT2016001) and Scientific Research Foundation for Young Academic Leaders in CUIT (J201415).
} 
influencing factors of the atmospheric pollution is important. In this paper, the influence of meteorological condition on the atmospheric pollutants $\left(\mathrm{PM}_{2.5}\right.$, $\mathrm{PM}_{10}, \mathrm{CO}, \mathrm{NO}_{2}$ and $\mathrm{SO}_{2}$ ) concentration and $\mathrm{AQI}$ index were studied based on the monitoring data $(28 / 10 / 2013 \sim 16 / 5 / 2014)$.

\section{Experimental Part}

\subsection{The influence of wind on atmospheric pollution in Chengdu}

\subsubsection{Wind direction}

Wind direction is an important factor for atmospheric pollution [3, 4]. The leading wind direction in Chengdu is the north wind all the year, so it is necessary to discuss the differences of main atmospheric pollutants in the south wind period and the north wind period. Ratio of all the pollutants concentrations in the south wind period and in the north wind were used to discuss the effect of wind direction on atmospheric pollution in Chengdu.

In the survey period, for the particulates concentration radio (the north wind period / the south wind period), there were $77.8 \%$ of the values less than $100 \%$, which concentrated in $40 \% \sim 100 \%$. This indicated the AQI and the average concentration of particulates in south wind period was higher than in the north wind period, which led to the more serious pollution. The influence trends of wind direction on the AQI index and the concentration of $\mathrm{PM}_{2.5}$ and $\mathrm{PM}_{10}$ were very similar. For AQI, the radio of the south wind period / the north wind less than $100 \%$ covers the $66.67 \%$ of the whole data. On the contrary, the radio of the north wind period / the south wind higher than $100 \%$ covered $33.33 \%$ of the whole data, and concentrated in $110 \%$. This showed that the pollution is worse when in the south wind period than the north. The AQI index of the southern cities of Chengdu including Ya an, Le shan, $\mathrm{Zi}$ yang, $\mathrm{Zi}$ gong and $\mathrm{Yi}$ bin higher than 160 covering about $70 \%$, of which higher than 200 covering $45 \%$. Instead, the north of Chengdu is Aba autonomous prefecture surrounded by Qiong Lai Mountain which could resist the diffusion of the atmospheric pollutants, and reduce the pollution of Chengdu in the north wind period. The north wind only took pollution air from Mian yang and De yang city which were located in the northeast of Chengdu. So the atmospheric pollution was more serious in the south wind period in Chengdu.

\subsubsection{Wind speed}

The wind speed is one of the main influencing factors of atmospheric pollutants $[3,4]$. The greater of wind speed, the faster of transmission of pollutants. Because of the wind in Chengdu is almost breeze which is lower than 3 levels, 
so the ratio is defined as the pollutants concentration with the wind higher than 3 4 levels / the pollutants concentration with breeze (lower than 3 levels).

The pollutants concentration ratio of the gale day / the three days before gale day all showed a raising tendency except $\mathrm{SO}_{2}$. For example, the concentration of $\mathrm{PM}_{2.5}, \mathrm{PM}_{10}, \mathrm{CO}$ and $\mathrm{NO}_{2}$ in the gale day on $18 / 03 / 2013$ was $1.09,1.04,1.11$ and 1.26 times as much as the concentrations in three days before. It suggested the gale brought more pollutants leading to the decrease of the atmospheric quality. The concentration of $\mathrm{SO}_{2}$ was declined in the gale day / the three days before gale day, suggested that gale not only take pollutants from outside to increase the pollution, but also diluted the pollutants.

\subsubsection{The contribution of local source and external source}

The value of local source was defined as the average concentration of pollutants in three breeze days before gale day. If the breeze period was less than 3 days, the existing days were selected. The contribution of external source was the $\mathrm{D}$-value of concentration in the gale period and in the breeze period. Results showed that the proportions of local source of $\mathrm{PM}_{2.5}, \mathrm{PM}_{10}, \mathrm{CO}$ and $\mathrm{NO}_{2}$ were $77.47 \%, 84.97 \%, 78.81 \%$ and $76.1 \%$, respectively. It indicated that pollutants were mainly from local source. In the gale period the wind is always from north, so the pollution in the north is more serious in Chengdu. Assumed that all the pollutants were discharged from local source in the breeze days, by which $30 \%$ air pollutants from external source were estimated taken by the south wind. It suggested that external source could not be ignored which can increase the atmospheric pollutants concentration in Chengdu.

\subsection{The influence of rainfall on the concentration of atmospheric pollutants}

\subsubsection{The clean-up effect of rainfall on the main atmospheric pollutants}

Generally speaking, the rainfall is an effective meteorological factor for cleaning the atmosphere [5]. To study the clean-up effect of rainfall on the pollutants, the days of rainfall during October 28, 2013 to May 16, 2014 were selected and the clean-up efficiency of the rainfall to the pollutants $\left(P_{0}\right)$ is calculated by formula.

$$
P_{0}=1-P_{1} / P_{2}
$$

In formula: $P_{0}$ - the efficiency of clean-up;

$P_{1}$ - The concentration of pollutants on the day after the rainfall;

$P_{2}$ - The concentration of pollutants on the day before the rainfall. 
The proportion of the clean-up efficiency of rainfall to the particulates over 0 is $87.5 \%$, which focus on about $25 \%$, while the efficiency of the rainfall to $\mathrm{PM}_{2.5}$ reached to $65 \%$ on March 20, 2014, because rainfall reached $4.9 \mathrm{~mm}$ on the day. This showed that the rainfall has a good cleaning effect to the particulates. But there were some days on which the particulates concentration was higher in the rainy day than the day before the rain day, such as December 24, 2013, January 27, 2014 and April 17, 2014, whose particulates concentration was 1.35 times, 1.35 times and 1.03 times as much as the day before the rain day. This situation could be explained by: (1) rain enriched particulate matter in the days before the rainy day; (2) the south wind on the rainy day brought with the pollutants from external source increased the pollutants in the rain day. The tend line of the effect of rainfall on the gas pollutants had the same tend with the particulates. The clearance efficiency was around $20 \%$, but the negative value of proportion covered $23.5 \%, 17.65 \%$ and $20.50 \%$ of $\mathrm{CO}, \mathrm{NO}_{2}$ and $\mathrm{SO}_{2}$. This situation was similar with the particulates pollution. The reason was also the same.

\subsubsection{Recovery radio of main atmospheric pollutants after rain}

The recovery radio of main atmospheric pollutants after rainfall is defined as the pollutants concentrations after the rainy day/ the pollutants concentrations before the rainy day. Results showed the recovery radio are lower than $100 \%$, especially the ratio for particulates is only $40 \sim 60 \%$. This suggested that the wash efficiency for particulates is obvious by rainfall, which can reach to $40 \%$ $60 \%$ and $60 \%$ at most.

\subsection{The influence of temperature on atmospheric pollution}

The AQI index in December, 2013 and April, 2014 representing the atmospheric pollution in winter and summer was selected to discuss the influence of temperature on atmospheric pollution. In general, the pollution was more serious in winter than in summer, the AQI index in winter was $2 \sim 4$ times as much as in summer. The related research also showed that temperature has some influence on atmospheric pollution.

\subsection{The influence of humidity on the atmospheric pollution}

Humidity has some influence on the atmospheric pollution. During the statistical period, particulates and $\mathrm{CO}$ had a good positive relativity. That is to say, the humidity higher, the concentration of particulates and $\mathrm{CO}$ is getting higher too. On the other side, no obvious relativity was observed between the humidity and $\mathrm{NO}_{2}, \mathrm{SO}_{2}$. No matter the humidity increase or decrease, the concentration $\mathrm{NO}_{2}$ 
$\left(20 \sim 100 \mu \mathrm{g} / \mathrm{m}^{3}\right)$ and $\mathrm{SO}_{2}\left(10 \sim 50 \mu \mathrm{g} / \mathrm{m}^{3}\right)$ were around $60 \mu \mathrm{g} / \mathrm{m}^{3}$ and $25 \mu \mathrm{g} / \mathrm{m}^{3}$. That suggested the influence of humidity on atmospheric ollution is mainly embodied in the pollution of the particulates and $\mathrm{CO}$.

\subsection{The influence of different weather on atmospheric pollution}

The tendency of concentration of pollutants during the overcast day is uncertainty, while the tendency of concentration increased in cloudy day and sunny day. The increasing ratio of the pollutants is smaller in sunny day than in cloudy day. This may be explained that pollutants can be enriched in clouds and its diffusion was hindered by clouds, resulting in a rapid increase in concentration in cloudy day. Taking two days (December 15, 2013 for cloudy day after rain and April 19, 2014 for overcast day after rain) for comparison, the concentration of particulates and gas pollutants decreased $56 \%$ and $30 \%$ on December 15, 2013, while the concentration of particulates and gas pollutants decreased $62 \%$ and $35 \%$ on April 19, 2014. That is to say, the concentration decreased more in cloudy day than in overcast day. Because deep clouds in cloudy would hinder the spread of the pollutants, while thin clouds in overcast day could be benefit for the dilution of the pollutants.

\section{Conclusions}

Comparative analysis of the influence of the meteorological conditions on the atmospheric pollutants concentration in Chengdu based on the statistical analysis of meteorological data of the main atmospheric pollutants including $\mathrm{PM}_{2.5}, \mathrm{PM}_{10}, \mathrm{CO}, \mathrm{NO}_{2}$ and $\mathrm{SO}_{2}$ showed that air pollution of Chengdu is more serious during south wind period than the north wind period. When the wind is strong, the concentration of all pollutants is on the rise except $\mathrm{SO}_{2}$. The wind bring external input source which accounted for $10 \% \sim 40 \%$. Rainfall clearance ratio of air pollutants can reach more than $60 \%$, and the particulate matter concentration can reduce $40 \% \sim 60 \%$. Temperature can affect the concentration of air pollutants, because the inversion layer in different seasons affects the diffusion of atmospheric pollutants. It is more conducive to pollutant diffusion on overcast days than cloudy weather.

\section{References}

1. Ma J, Xu X, Zhao C, et al. A Review of Atmospheric Chemistry Research in China:Photochemical Smog, Haze Pollution,and Gas-Aerosol Interactions [J]. Advances in Atmospheric Sciences, 2012, 29(5):1006-1026

2. Tao J, Cheng T, Zhang R, et al. Chemical Composition of PM2.5 at an Urban Site of Chengdu in Southwestern China [J]. Advances in 
Atmospheric Sciences, 2013, 30(4):1070-1084

3. Zhao Chen-xi, Wang Yun-qi, Wang Yu-jie, et al. Temporal and Spatial Distribution of PM2.5 and Pollution Status and the Correlation of Particulate Matters and Meteorological Factors During Winter and Spring in Beijing[J]. Environmental Science, 2014, 02(35):418-427. in Chinese

4. Sui Ke-ke, Wang Zi-fa, Yang Jun, et al. Beijing Persistent PM10 Pollution and its Relationship with General Meteorological Features [J]. Research of Environmental Sciences, 2007, 06(20):77-82. in Chinese

5. Feng Jian-jun, Shen Jia-fen, Liang Ren-zhong, et al. Analysis of Relationship of Meteorologital Elements and PM10 in Guangzhou [J]. Environmental Monitoring in China, 2009, 01(25):78-82. in Chinese 